\title{
Galaxy disc heating as a result of minor mergers
}

\author{
M. T. Tapia ${ }^{1}$, M. Balcells ${ }^{1}$ and M. C. Eliche-Moral ${ }^{2}$ \\ ${ }^{1}$ Instituto de Astrofísica de Canarias, Vía Láctea S/N, \\ E-38205 La Laguna, Tenerife, Spain \\ email: ttapia@iac.es \\ ${ }^{2}$ Departamento de Astrofísica y Ciencias de la Atmósfera, Facultad de C.C. Físicas, \\ Universidad Complutense de Madrid, 28040 Madrid, Spain \\ email: cem@astrax.fis.ucm.es
}

\begin{abstract}
We perform a sample of N-body simulations of a minor merger between a Milky Way type galaxy and a satellite, which is density scaled version of the primary galaxy. In this suite of collisionless runs we change the value of some critical parameters like the luminous mass ratio between the two galaxies $(1: 6,1: 9)$, the type of orbit (direct or prograde) and the number of particles $(185,000,555,000$ and $1,850,000)$. We estimate the disc thickening by measuring the median of the vertical scale and we find that that the merger increases the scale height in a factor of $\approx 2$ in all the remnants.
\end{abstract}

Keywords. methods: n-body simulations,galaxies: interactions, galaxies: structure

\section{Introduction}

A lot of numerical n-body simulations have been done during the last decades to study the effect of the minor mergers in the galactics disc with great detail (Quinn et al. 1993, Hernquist \& Mihos 1995). One of the main conclusions of these studies is that the fusion processes in the range of $M_{\mathrm{sat}} / M_{\text {disk }} \approx 0.05-0.2$ can cause a vertical grow of the disc in a factor between 2 and 4, depending to the distance at the galactic centre. Since it is still an open question the relevance of the minor mergers in the grow of the galactic disc, is very important to give better measurements of the disc thickening due to the satellite acretion.

\section{Models and Results}

We build a Milky Way type galaxy with the GalactICS code (Kuijken \& Dubinski 2005, hereafter KD05) which consist in an exponential disk, a King bulge and a dark halo. The parameters of the model are the same as the called "A" by them. The satellite is a reply density scaled of the primary as is it described in González-García \& Balcells (GonzalezGarcia \& Balcells 2006). We use three different number of particles $(185,000,555,000$, 1850, 000), 2 luminous mass ratio and encounter orbits (tab. 1). The 12 final models were executed in LaPalma cluster and we let it evolve during $\sim 3$ Gyr and $\sim 4$ Gyr for the luminous mass ratio of $1 / 6$ and $1 / 9$, respectively. To assign a name to the models we use the next nomenclature: $\mathbf{M} \alpha \mathbf{N} \beta \gamma$, where $\mathbf{M}$ means model, $\alpha$ the initial luminous mass ratio between the satellite and the bulge of the primary, $\mathbf{N} \beta$ the number of particles and $\gamma$ if the fusion is direct $(\mathrm{D})$ o retrograde $(\mathrm{R})$.

We measure the median distribution of the particles weighted by the particle mass at four fixed radial positions $(1 / 10,1 / 3,1 / 2,1)$ of the radial length, each one with a width of 
Table 1. Scale and orbital paramateres of the models

\begin{tabular}{cccccccccc}
\hline $\begin{array}{c}G_{\text {modelo }} \\
(1)\end{array}$ & $\begin{array}{c}M R_{1} \\
(2)\end{array}$ & $\begin{array}{c}M R_{2} \\
(3)\end{array}$ & $\begin{array}{c}R_{S} / R_{P} \\
(4)\end{array}$ & $\begin{array}{c}L_{Z, 0} \\
(5)\end{array}$ & $\begin{array}{c}e \\
(6)\end{array}$ & $\begin{array}{c}R_{\text {peri }} \\
(7)\end{array}$ & $\begin{array}{c}V_{\text {peri }} \\
(8)\end{array}$ & $\begin{array}{c}\theta_{1} \\
(9)\end{array}$ & $\begin{array}{c}t_{\text {tot }} \\
(10)\end{array}$ \\
\hline M2TF35D & $1 / 2$ & $1 / 6$ & 0.46 & 0.4815 & 0.907 & 0.73 & 1.981 & 30 & 150 \\
M2TF35R & $1 / 2$ & $1 / 6$ & 0.46 & 0.4815 & 0.907 & 0.73 & 1.981 & 150 & 150 \\
M3TF35D & $1 / 3$ & $1 / 9$ & 0.39 & 0.3532 & 0.900 & 0.79 & 1.793 & 30 & 200 \\
M3TF35R & $1 / 3$ & $1 / 9$ & 0.39 & 0.3532 & 0.900 & 0.79 & 1.793 & 150 & 200
\end{tabular}

col.(1):model group, col.(2):satellite luminous mass ratio between the satellite and the bulge of the primary, col.(3):initial luminous mass ratio, col.(4):initial half mass radius ratio, col.(5): initial orbital angular moment, col.(6):orbit excentricity, col.(7):pericenter distance, col.(8): pericenter velocity, col.(9): initial angle between the orbit angular moment and the disc spin of the primary,col.(10): total timesteps in simulation units.

$3 \%$ (Fig. 1). To estimate and correct our measurements by the numerical heating of the disc, we did in the same way as Eliche-Moral et al. 2006 (hereafter EM06).

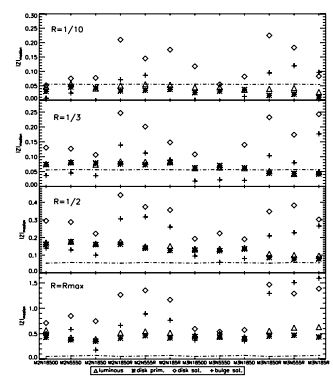

Figure 1. Median of the vertical distribution of the final remants separated by model, radial position and component. The triangles are all the luminous material, the asterisks to the primary disc, the diamonds to the satellite disk and the cruises the satellite bulge. These measurements are corrected by the numerical thickening of the disc.

\section{Conclusion}

The numerical thickening of the disk depend of the number of particles, our 185, 000 particles models have an average value of 0.019 , the 555, 000 of 0.0064 and the 1, 8500,000 of 0.0062 in close agreement with KD05 and EM06. After correct our models, all of them have a vertical scale $\approx 2$. We find that there is not a clear relation between the model and the value of the median at different radial positions, so the disc growth is most likely due to global instabilities than two-body effect.

\section{References}

González-García, A. C. \& Balcells, M. 2005, MNRAS, 357, 735

Eliche-Moral, M. C., Balcells M., Aguerri, A. L., \& González-García, A. C. 2006, AA, 457, 91

Kuijken, K. \& Dubinski, J. 1995, MNRAS, 277, 1341

Kuijken, K. \& Dubinski, J. 1994, MNRAS, 269, 13

Toomre, A. \& Toomre, J. 1972, ApJ, 178, 623

Springel, V. 2005, MNRAS, 364, 1105

Hernquist, L. \& Mihos, J. C. 1995, ApJ, 448, 41

Quinn, P. J., Hernquist, L., \& Fullagar, D. P. 1993, AJ, 403, 74,

Tóth, G. \& Ostriker, J. P. 1992, ApJ, 389, 5

Zaritsky, D. 1995, ApJ, 448, L17

Hernquist, L. \& Katz, N. 1989, ApJS, 70, 419 